Publ. Mat. 60 (2016), 565-582

DOI: 10.5565 /PUBLMAT_60216_10

\title{
COMMON ZEROS PRESERVING MAPS ON VECTOR-VALUED FUNCTION SPACES AND BANACH MODULES
}

\author{
Maliheh Hosseini And Fereshteh SAdy
}

\begin{abstract}
Let $X, Y$ be Hausdorff topological spaces, and let $E$ and $F$ be Hausdorff topological vector spaces. For certain subspaces $A(X, E)$ and $A(Y, F)$ of $C(X, E)$ and $C(Y, F)$ respectively (including the spaces of Lipschitz functions), we characterize surjections $S, T: A(X, E) \rightarrow A(Y, F)$, not assumed to be linear, which jointly preserve common zeros in the sense that $Z\left(f-f^{\prime}\right) \cap Z\left(g-g^{\prime}\right) \neq \emptyset$ if and only if $Z\left(S f-S f^{\prime}\right) \cap$ $Z\left(T g-T g^{\prime}\right) \neq \emptyset$ for all $f, f^{\prime}, g, g^{\prime} \in A(X, E)$. Here $Z(\cdot)$ denotes the zero set of a function. Using the notion of point multipliers we extend the notion of zero set for the elements of a Banach module and give a representation for surjective linear maps which jointly preserve common zeros in module case.
\end{abstract}

2010 Mathematics Subject Classification: Primary: 46J10, 47B48; Secondary: 46J20.

Key words: Non-vanishing functions, Banach modules, maps preserving common zeros, vector-valued continuous function, point multipliers, zero set.

\section{Introduction}

The study of linear maps preserving some properties related to the elements of spaces under consideration is one of the most active areas of research in recent years. Characterization of linear maps preserving common zeros between vector-valued function spaces is one of these problems which may be considered as a vector-valued version of the Banach-Stone and the Gleason-Kahane-Żelasko theorems. These maps send (in two directions) every pair of functions with disjoint zero sets to functions with the same property. For completely regular spaces $X, Y$, Hausdorff topological vector spaces $E, F$, and subspaces $A(X, E)$ and $A(Y, F)$ of $C(X, E)$ and $C(Y, F)$, respectively, a characterization of linear bijections $T: A(X, E) \rightarrow A(Y, F)$ satisfying

$$
\bigcap_{i=1}^{n} Z\left(f_{i}\right) \neq \emptyset \Longleftrightarrow \bigcap_{i=1}^{n} Z\left(T f_{i}\right) \neq \emptyset,
$$


for any $n \in \mathbb{N}$ and $f_{1}, \ldots, f_{n} \in A(X, E)$, has been given in [17]. Here $Z(\cdot)$ denotes the zero set of a function. In the case where $X, Y$ are metric spaces and $E, F$ are normed spaces, a complete description of linear bijections $T: A(X, E) \rightarrow A(Y, F)$ between certain subspaces of $C(X, E)$ and $C(Y, F)$, satisfying the weaker condition

$$
Z(f) \cap Z(g) \neq \emptyset \Longleftrightarrow Z(T f) \cap Z(T g) \neq \emptyset,
$$

for all $f, g \in A(X, E)$, is given in [7] and then some extensions of the previous results are obtained. In [18], among other things, the authors considered maps preserving zero set containments, which dates back to [11]. Indeed, they characterized linear bijections $T: C(X, E) \rightarrow C(Y, F)$ such that

$$
Z(f) \subseteq Z(g) \Longleftrightarrow Z(T f) \subseteq Z(T g),
$$

when $X, Y$ are either realcompact or metric spaces, and $E, F$ are locally convex spaces. Moreover, it is worth mentioning that another related notion, originally introduced in [18], is non-vanishing preserving maps in the sense that

$$
Z(f)=\emptyset \Longleftrightarrow Z(T f)=\emptyset .
$$

For some results concerning non-vanishing preserving maps (on lattices) one can see $[\mathbf{4}, \mathbf{6}, \mathbf{5}, \mathbf{8}, \mathbf{9}, \mathbf{1 5}, \mathbf{1 8}, \mathbf{1 9}, \mathbf{2 0}]$. We also refer to $[\mathbf{1 4}]$ for some relevant concepts in the case of scalar-valued continuous functions.

In this paper we consider a pair of maps, not assumed to be linear, jointly preserving common zeros in the sense which is defined below. Let $X, Y$ be Hausdorff topological spaces, $E, F$ be Hausdorff topological vector spaces, and $A(X, E)$ and $A(Y, F)$ be subspaces of $C(X, E)$ and $C(Y, F)$, respectively. We say that a pair of maps $S, T: A(X, E) \rightarrow$ $A(Y, F)$, not assumed to be linear, jointly preserves common zeros if

$$
Z\left(f-f^{\prime}\right) \cap Z\left(g-g^{\prime}\right) \neq \emptyset \Longleftrightarrow Z\left(S f-S f^{\prime}\right) \cap Z\left(T g-T g^{\prime}\right) \neq \emptyset
$$

for all $f, f^{\prime}, g, g^{\prime} \in A(X, E)$. A map $T: A(X, E) \rightarrow A(Y, F)$ preserves common zeros if the pair $T, T$ jointly preserves common zeros. In Section 3 , we study such maps for certain subspaces of vector-valued continuous functions on Hausdorff spaces with values in a Hausdorff topological vector space and obtain generalizations of the results in [7]. In particular, for the spaces of Lipschitz functions we give an extension of $[\mathbf{5}$, Theorem 6]. In Section 4 we define the zero set for the elements of Banach modules and consider linear surjective maps which jointly preserve common zeros between Banach modules. We show that such maps admit representations similar to weighted composition operators. 


\section{Preliminaries}

Let $X$ be a Hausdorff topological space and let $E$ be a Hausdorff topological vector space. We denote the space of all $E$-valued continuous functions on $X$ by $C(X, E)$ and we set $C(X)=C(X, \mathbb{C})$.

A subspace $A(X, E)$ of $C(X, E)$ is said to be completely regular if for each $x \in X$ and each closed subset $C$ of $X$ such that $x \notin C$, there exists $f \in A(X, E)$ with $f(x) \neq 0$ and $f=0$ on $C$.

For a bounded metric space $(X, d), \alpha \in(0,1]$, and a Banach space $E$, let $\operatorname{Lip}_{\alpha}^{b}(X, E)$ be the space of all $E$-valued $\alpha$-Lipschitz functions $f: X \rightarrow$ $E$, that is

$$
L_{\alpha}(f)=\sup _{\substack{x, x^{\prime} \in X \\ x \neq x^{\prime}}} \frac{\left\|f(x)-f\left(x^{\prime}\right)\right\|}{d^{\alpha}\left(x, x^{\prime}\right)}<\infty
$$

and we set $\operatorname{Lip}_{\alpha}^{b}(X)=\operatorname{Lip}_{\alpha}^{b}(X, \mathbb{C})$. Then $\operatorname{Lip}_{\alpha}^{b}(X, E)$ is a Banach space under the following norm

$$
\|f\|=\max \left\{L_{\alpha}(f),\|f\|_{\infty}\right\} \quad\left(f \in \operatorname{Lip}_{\alpha}^{b}(X, E)\right),
$$

where $\|\cdot\|_{\infty}$ denotes the supremum norm. As usual, for $\alpha \in(0,1)$, $\operatorname{lip}_{\alpha}^{b}(X, E)$ denotes the Banach space of those functions $f \in \operatorname{Lip}_{\alpha}^{b}(X, E)$ with $\lim _{d\left(x, x^{\prime}\right) \rightarrow 0} \frac{\left\|f(x)-f\left(x^{\prime}\right)\right\|}{d^{\alpha}\left(x, x^{\prime}\right)}=0$ and we use the notation $\operatorname{lip}_{\alpha}^{b}(X)$ for $\operatorname{lip}_{\alpha}^{b}(X, \mathbb{C})$ which is, indeed, a commutative unital Banach algebra. Clearly

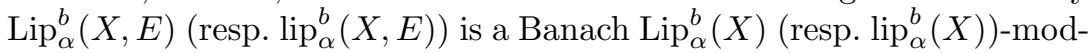
ule.

For a Banach algebra $\mathcal{A}$ with non-empty character space $\sigma(\mathcal{A})$, by $\sigma(\mathcal{A}) \cup\{0\}$ we mean the character space of the unitization of $\mathcal{A}$. Let $\mathcal{X}$ be a Banach left $\mathcal{A}$-module. Following [3], for a point $\varphi \in \sigma(\mathcal{A}) \cup\{0\}$, a linear functional $\xi \in \mathcal{X}^{*}$ is said to be a point multiplier at $\varphi$ if $\langle\xi, a$. $x\rangle=\varphi(a)\langle\xi, x\rangle$ for all $a \in \mathcal{A}$ and $x \in \mathcal{X}$. The submodules of $\mathcal{X}$ with codimension one will be referred to as hyper maximal (left) submodules of $\mathcal{X}$. It is easy to see that the kernel of each point multiplier on $\mathcal{X}$ is a closed hyper maximal submodule of $\mathcal{X}$ and conversely, each closed hyper maximal submodule of $\mathcal{X}$ is the kernel of some non-trivial point multiplier on $\mathcal{X}$ (see for example [3] for the unital case).

We denote the set of all non-trivial point multipliers on $\mathcal{X}$ which are in the unit ball of $X^{*}$ by $\sigma_{\mathcal{A}}(\mathcal{X})$ and the set of all closed hyper maximal submodules of $\mathcal{X}$ by $\Delta_{\mathcal{A}}(\mathcal{X})$. Then $\nu_{\mathcal{A}}: \sigma_{\mathcal{A}}(\mathcal{X}) \rightarrow \sigma(\mathcal{A}) \cup\{0\}$ is the natural map which associates to each point multiplier $\xi$ on $\mathcal{X}$, the unique point $\varphi \in \sigma(\mathcal{A}) \cup\{0\}$ satisfying $\langle\xi, a \cdot x\rangle=\varphi(a)\langle\xi, x\rangle$ for all $a \in \mathcal{A}$ and $x \in \mathcal{X}$. The Gelfand radical $\operatorname{Rad}_{\mathcal{A}}(\mathcal{X})$ of $\mathcal{X}$ is the intersection 
$\cap_{\varphi \in \sigma(\mathcal{A}) \cup\{0\}} \mathcal{X}_{\varphi}$, where for each $\varphi \in \sigma(\mathcal{A}) \cup\{0\}, \mathcal{X}_{\varphi}$ is the closure of the set $\left\{\sum_{k=1}^{n} a_{k} \cdot x_{k}: a_{k} \in \mathcal{A}, x_{k} \in \operatorname{ker} \varphi\right\}$. Indeed, by [3, Proposition 3.2(i)], $\operatorname{Rad}_{\mathcal{A}}(\mathcal{X})=\cap_{\xi \in \sigma_{\mathcal{A}}(\mathcal{X})} \operatorname{ker}(\xi)$. We say that $\mathcal{X}$ is hyper semisimple if $\operatorname{Rad}_{\mathcal{A}}(\mathcal{X})=\{0\}$.

Consider the following subset of $\Pi_{P \in \Delta_{\mathcal{A}}(\mathcal{X})} \mathcal{X} / P$ :

$$
\underline{\mathcal{X}}=\left\{\underline{x}=\left(x_{P}+P\right)_{P \in \Delta_{\mathcal{A}}(\mathcal{X})}: \sup _{P \in \Delta_{\mathcal{A}}(\mathcal{X})}\left\|x_{P}+P\right\|<\infty\right\} .
$$

Then $\underline{\mathcal{X}}$ is a Banach space under the norm defined by

$$
\|\underline{x}\|=\sup _{P \in \Delta_{\mathcal{A}}(\mathcal{X})}\left\|x_{P}+P\right\|, \quad \underline{x}=\left(x_{P}+P\right)_{P \in \Delta_{\mathcal{A}}(\mathcal{X})} \in \underline{\mathcal{X}},
$$

which is actually a left Banach $\mathcal{A}$-module in a natural way (see $[\mathbf{3}]$ for the case that $\mathcal{A}$ is unital). Furthermore, the map $G_{\mathcal{X}}: \mathcal{X} \rightarrow \underline{\mathcal{X}}$ defined by $G_{\mathcal{X}}(x)=\widehat{x}$, where for each $x \in \mathcal{X}, \widehat{x}=(x+P)_{P \in \Delta_{\mathcal{A}}(\mathcal{X})}$ is a norm decreasing map which is injective if $\mathcal{X}$ is hyper semisimple.

\section{Jointly common zeros preserving maps between certain subspaces of vector-valued functions}

In the main theorem of this section (Theorem 3.1), we assume that $X, Y$ are Hausdorff topological spaces, $E, F$ are Hausdorff topological vector spaces, and $A(X, E)$ and $A(Y, F)$ are subspaces of $C(X, E)$ and $C(Y, F)$, respectively, satisfying the following property:

(Z) For each $x \in X$ and $y \in Y$ and neighborhoods $U$ and $V$ of $x$ and $y$, respectively, there are functions $f \in A(X, E)$ and $g \in A(Y, F)$ with $x \in Z(f) \subseteq U$ and $y \in Z(g) \subseteq V$.

We recall that a pair of, not necessarily linear, maps $S, T: A(X, E) \rightarrow$ $A(Y, F)$ jointly preserve common zeros if for any $f, f^{\prime}, g, g^{\prime} \in A(X, E)$,

$$
Z\left(f-f^{\prime}\right) \cap Z\left(g-g^{\prime}\right) \neq \emptyset \Longleftrightarrow Z\left(S f-S f^{\prime}\right) \cap Z\left(T g-T g^{\prime}\right) \neq \emptyset .
$$

Clearly under the additional assumption $S 0=T 0=0$, such maps preserve non-vanishing functions in the sense that for each $f \in A(X, E)$,

$$
\begin{aligned}
& Z(f) \neq \emptyset \Longleftrightarrow Z(S f) \neq \emptyset, \\
& Z(f) \neq \emptyset \Longleftrightarrow Z(T f) \neq \emptyset .
\end{aligned}
$$

A point $x \in X$ is called a zero point of $A(X, E)$ if $Z(f)=\{x\}$ for some $f \in A(X, E)$. 
Theorem 3.1. Let $A(X, E), A(Y, F)$ satisfy property $(\mathrm{Z})$, and let $S, T: A(X, E) \rightarrow A(Y, F)$ be surjective, not necessarily linear, maps. If $S, T$ jointly preserve common zeros, then there exist subsets $X_{0}$ and $Y_{0}$ of $X$ and $Y$ respectively, containing all zero points of $A(X, E)$ and $A(Y, F)$ respectively, a function $h: Y_{0} \rightarrow X_{0}$ (which is a homeomorphism whenever $A(X, E), A(Y, F)$ are completely regular $)$, subspaces $E_{y} \subseteq E$, $F_{y} \subseteq F$, and bijections $J_{y}: E_{y} \rightarrow F_{y}, G_{y}: E_{y} \rightarrow F_{y}$ for each $y \in Y_{0}$, such that

$$
S f(y)=S 0(y)+J_{y}(f(h(y))), \quad T f(y)=T 0(y)+G_{y}(f(h(y)))
$$

for all $f \in A(X, E)$ and $y \in Y_{0}$. If furthermore $S, T$ are linear, then for all $y \in Y_{0}, J_{y}$, and $G_{y}$ are linear.

Proof: Since the pair $S-S 0$ and $T-T 0$ jointly preserves common zeros, as well, without loss of generality we may assume that $S 0=T 0=0$.

We prove the theorem through the following steps:

Step 1: $S$ and $T$ are injective maps.

Since the conditions are symmetric with respect to $S$ and $T$, it suffices to show that $T$ is injective. Let $f, f^{\prime} \in A(X, E)$ with $T f=T f^{\prime}$. For each $x \in X$ and neighborhood $U$ of $x$ there exists, by property $(\mathrm{Z})$, a function $f_{0} \in A(X, E)$ with $x \in Z\left(f_{0}\right) \subseteq U$. Clearly, $Z\left(T f-T f^{\prime}\right) \cap Z\left(S f_{0}\right) \neq \emptyset$, which implies that $Z\left(f-f^{\prime}\right) \cap Z\left(f_{0}\right) \neq \emptyset$. Hence $Z\left(f-f^{\prime}\right) \cap U \neq \emptyset$ and therefore $x \in Z\left(f-f^{\prime}\right)$, since $Z\left(f-f^{\prime}\right)$ is closed. Thus $f=f^{\prime}$ and consequently $T$ is injective.

For each $x \in X$, we set $\mathcal{I}_{x}(S)=\cap_{x \in Z\left(f-f^{\prime}\right)} Z\left(S f-S f^{\prime}\right)$ and similarly $\mathcal{I}_{x}(T)=\cap_{x \in Z\left(f-f^{\prime}\right)} Z\left(T f-T f^{\prime}\right)$.

Step 2: Given $x \in X$, the intersections $\mathcal{I}_{x}(S)$ and $\mathcal{I}_{x}(T)$ are singleton and equal whenever both are non-empty.

We show that if $y_{1} \in \mathcal{I}_{x}(S)$ and $y_{2} \in \mathcal{I}_{x}(T)$ then $y_{1}=y_{2}$. Assume on the contrary that $y_{1} \neq y_{2}$ and let $V_{1}, V_{2}$ be disjoint neighborhoods of $y_{1}$ and $y_{2}$ in $Y$, respectively. Then, by property $(\mathrm{Z})$, there are elements $l, g \in A(Y, F)$ such that $y_{1} \in Z(l) \subseteq V_{1}$ and $y_{2} \in Z(g) \subseteq V_{2}$. In particular, $Z(l) \cap Z(g)=\emptyset$ and hence $Z\left(T^{-1} l\right) \cap Z\left(S^{-1} g\right)=\emptyset$. Given a neighborhood $U$ of $x$, let $f \in A(X, E)$ be such that $x \in Z(f) \subseteq U$. Then since $y_{2} \in Z(g) \cap Z(T f)$ it follows that $Z\left(S^{-1} g\right) \cap Z(f) \neq \emptyset$. Therefore, $Z\left(S^{-1} g\right) \cap U \neq \emptyset$ and since $U$ is arbitrary, the closedness of $Z\left(S^{-1} g\right)$ implies that $x \in Z\left(S^{-1} g\right)$. A similar discussion shows that $x \in Z\left(T^{-1} l\right)$, a contradiction. 
Clearly the above argument shows that two intersections are the same and singleton whenever both are nonempty.

We now consider the subset

$$
X_{0}:=\left\{x \in X: \mathcal{I}_{x}(S) \neq \emptyset, \mathcal{I}_{x}(T) \neq \emptyset\right\}
$$

of $X$. By Step 2, for each $x \in X_{0}$ there exists a unique point $y \in$ $Y$ such that $\mathcal{I}_{x}(S)=\mathcal{I}_{x}(T)=\{y\}$. Hence we can define a function $k: X_{0} \rightarrow Y$ such that for each $x \in X_{0}, k(x)$ is the unique point in the above intersections, that is $\mathcal{I}_{x}(S)=\mathcal{I}_{x}(T)=\{k(x)\}$. Similarly, we set $Y_{0}:=\left\{y \in Y: \mathcal{I}_{y}\left(S^{-1}\right) \neq \emptyset, \mathcal{I}_{y}\left(T^{-1}\right) \neq \emptyset\right\}$ and define a function $h: Y_{0} \rightarrow X$ such that for each $y \in Y_{0}, \mathcal{I}_{y}\left(S^{-1}\right)=\mathcal{I}_{y}\left(T^{-1}\right)=\{h(y)\}$.

Step 3: $k$ is a bijective map from $X_{0}$ onto $Y_{0}$ and $h=k^{-1}$. If furthermore $A(X, E)$ (resp. $A(Y, F)$ ) is completely regular, then $h$ (resp. $k$ ) is continuous.

Given $x \in X_{0}$ it suffices to show that $\mathcal{I}_{k(x)}\left(S^{-1}\right)=\mathcal{I}_{k(x)}\left(T^{-1}\right)=\{x\}$ which clearly implies that $k(x) \in Y_{0}$ and $h(k(x))=x$. For this purpose, suppose that $g, g^{\prime} \in A(Y, F)$ and $\left(g-g^{\prime}\right)(k(x))=0$. Let $U$ be an arbitrary neighborhood of $x$ and $f \in A(X, E)$ be chosen such that $x \in Z(f) \subseteq U$. Since $k(x) \in Z\left(g-g^{\prime}\right) \cap Z(T f)$ it follows that $Z\left(S^{-1} g-S^{-1} g^{\prime}\right) \cap Z(f) \neq \emptyset$, and so $Z\left(S^{-1} g-S^{-1} g^{\prime}\right) \cap U \neq \emptyset$ implying that $x \in Z\left(S^{-1} g-S^{-1} g^{\prime}\right)$. Similarly, $x \in Z\left(T^{-1} g-T^{-1} g^{\prime}\right)$. Therefore $\mathcal{I}_{k(x)}\left(S^{-1}\right)=\mathcal{I}_{k(x)}\left(T^{-1}\right)=$ $\{x\}$ and consequently $k(x) \in Y_{0}$ and $x=h(k(x))$.

A similar argument shows that for each $y \in Y_{0}, \mathcal{I}_{h(y)}(S)=\mathcal{I}_{h(y)}(T)=$ $\{y\}$, that is $k(h(y))=y$.

Assume now that $A(X, E)$ is completely regular. To prove the continuity of $h$, assume on the contrary that there exists a net $\left(y_{\alpha}\right)$ in $Y_{0}$ such that $y_{\alpha} \rightarrow y$, for some $y \in Y_{0}$ while $h\left(y_{\alpha}\right)$ does not converge to $h(y)$. Then there exists a subnet $\left(h\left(y_{\beta}\right)\right)$ of $\left(h\left(y_{\alpha}\right)\right)$ such that $h\left(y_{\beta}\right) \in X \backslash U$ for some neighborhood $U$ of $h(y)$. Using the complete regularity of $A(X, E)$, we can find a function $f \in A(X, E)$ such that $f\left(h\left(y_{\beta}\right)\right)=0$ for each $\beta$ while $f(h(y)) \neq 0$. Thus $S f\left(y_{\beta}\right)=S f\left(k\left(h\left(y_{\beta}\right)\right)\right)=0$ and consequently, $S f(y)=0$, since $S f$ is continuous. Hence $f(h(y))=0$, a contradiction. This argument shows that $h$ is continuous.

Similarly, $k$ is continuous, whenever $A(Y, F)$ is completely regular.

Now for each $y \in Y_{0}$ we consider the non-trivial subspaces $E_{y}:=$ $\{f(h(y)): f \in A(X, E)\}$ and $F_{y}:=\{g(y): g \in A(Y, F)\}$ of $E$ and $F$, respectively. We also define the maps $J_{y}: E_{y} \rightarrow F_{y}$ and $G_{y}: E_{y} \rightarrow F_{y}$ by 
$J_{y}(e)=S f(y)$ and $G_{y}(e)=T f(y)$ for all $e \in E_{y}$, where $f \in A(X, E)$ is such that $f(h(y))=e$. It is easy to see that $J_{y}$ and $G_{y}$ are well defined.

Step 4: For each $y \in Y_{0}, J_{y}$ and $G_{y}$ are bijective maps.

For the injectivity, assume that $e, e^{\prime} \in E_{y}$ and $J_{y}(e)=J_{y}\left(e^{\prime}\right)$. Then $S f(y)=S f^{\prime}(y)$ for all $f, f^{\prime} \in A(X, E)$ with $f(h(y))=e$ and $f^{\prime}(h(y))=e^{\prime}$. Therefore, by the definition of $h(y),\left(f-f^{\prime}\right)(h(y))=0$ since $(S f-$ $\left.S f^{\prime}\right)(y)=0$, that is $e=e^{\prime}$. Now we show that $J_{y}$ is surjective. For this suppose that $\mathrm{f} \in F_{y}$. Then there is an $f \in A(X, E)$ such that $S f(y)=$ f. So putting $e=f(h(y))$ we have $e \in E_{y}$ and $J_{y}(e)=S f(y)=\mathrm{f}$. Similarly, $G_{y}$ also is a bijective map.

Clearly, for each $y \in Y_{0}, J_{y}, G_{y}$ are linear whenever $S$ and $T$ are linear.

Step 5: $X_{0}$ (resp. $\left.Y_{0}\right)$ contains the zero points of $A(X, E)$ (resp. $A(Y, F)$ ). Indeed, if $x_{0} \in X$ such that $Z\left(f_{0}\right)=\left\{x_{0}\right\}$ for some $f_{0} \in A(X, E)$, then $x_{0} \in X_{0}$ and $Z\left(S f_{0}\right)=Z\left(T f_{0}\right)=\left\{k\left(x_{0}\right)\right\}$.

We apply a similar argument as the one given in Step 2. Clearly $Z\left(S f_{0}\right) \neq \emptyset$. Assume that $y_{1}, y_{2} \in Y$ are distinct points in $Z\left(S f_{0}\right)$. Let $g$ and $l$ be defined as in Step 2. Then $Z\left(T^{-1} g\right) \cap Z\left(f_{0}\right) \neq \emptyset$ and so since $Z\left(f_{0}\right)=\left\{x_{0}\right\}$ we conclude that $x_{0} \in Z\left(T^{-1} g\right)$. Similarly, $x_{0} \in Z\left(T^{-1} l\right)$ since $y_{1} \in Z(l) \cap Z\left(S f_{0}\right)$. Therefore $Z\left(T^{-1} g\right) \cap Z\left(T^{-1} l\right) \neq \emptyset$ and so $Z(g) \cap Z(l) \neq \emptyset$, which is impossible. Therefore $Z\left(S f_{0}\right)$ is a singleton $\left\{y_{0}\right\}$ for some $y_{0} \in Y$.

Suppose now that $f, f^{\prime} \in A(X, E)$ with $x_{0} \in Z\left(f-f^{\prime}\right)$. Evidently, $Z\left(S f-S f^{\prime}\right) \cap Z\left(T f_{0}\right) \neq \emptyset$ and $Z\left(T f-T f^{\prime}\right) \cap Z\left(S f_{0}\right) \neq \emptyset$, i.e. $y_{0} \in$ $Z\left(T f-T f^{\prime}\right) \cap Z\left(S f-S f^{\prime}\right)$. Hence we deduce that $x \in X_{0}$ and $Z\left(S f_{0}\right)=$ $Z\left(T f_{0}\right)=\left\{k\left(x_{0}\right)\right\}$.

Notice that in the theorem above, if $A(X, E)$ and $A(Y, F)$ contain the constant functions, then $E_{y}=E$ and $F_{y}=F$ for each $y \in Y_{0}$.

Remark. Using the theorem above for the case where $S=T$, we conclude that each surjective, not necessarily linear, map $T: A(X, E) \rightarrow A(Y, F)$ preserving common zeros has the same description given in this theorem. In particular, if $T$ is linear and all points in $X$ and $Y$ are zero points of $A(X, E)$ and $A(Y, F)$, respectively, then for each $f \in A(X, E)$ and $y \in Y$ we have $T f(y)=G_{y}\left(f(h(y))\right.$, where $G_{y}: E_{y} \rightarrow F_{y}$ is a bijective linear map. So our result is an extension of [7, Theorem 3.1] and may be compared with some recent results such as $[\mathbf{1 7}$, Theorem 10] and $[\mathbf{1 8}$, Theorem 4.4 (Case 3)]. 
Corollary 3.2. Under the hypotheses of Theorem 3.1, if $A(X, E)$, $A(Y, F)$ are completely regular and all points in $X$ and $Y$ are zero points for $A(X, E)$ and $A(Y, F)$ respectively, then there exist a homeomorphism $h: Y \rightarrow X$, subspaces $E_{y} \subseteq E, F_{y} \subseteq F$, and bijections $J_{y}, G_{y}: E_{y} \rightarrow F_{y}$, $y \in Y$, such that

$$
S f(y)=S 0(y)+J_{y}(f(h(y))), \quad T f(y)=T 0(y)+G_{y}(f(h(y)))
$$

for all $f \in A(X, E)$ and $y \in Y$.

According to [7, Lemma 2.3], if $E$ and $F$ are vector lattices, $A(X, E)$ and $A(Y, F)$ are vector sublattices of $C(X, E)$ and $C(Y, F)$, then for any vector lattice isomorphism $S: A(X, E) \rightarrow A(Y, F)$ we have $\cap_{i=1}^{n} Z\left(f_{i}\right) \neq$ $\emptyset$ if and only if $\cap_{i=1}^{n} Z\left(S f_{i}\right) \neq \emptyset$ for all $n \in \mathbb{N}$ and $f_{1}, \ldots, f_{n} \in A(X, E)$. So the next corollary is a generalization of [5, Theorem 6].

Corollary 3.3. Let $X$ and $Y$ be bounded metric spaces, $E$ and $F$ be Banach spaces. Assume, further, that either $Y$ has no isolated points, or one of $E$ and $F$ has finite dimension. If $T, S: \operatorname{Lip}_{\alpha}^{b}(X, E) \rightarrow \operatorname{Lip}_{\alpha}^{b}(Y, F)$, $0<\alpha \leq 1$, are additive (resp. linear) surjections jointly preserving common zeros, then $T, S$ are continuous and there exist an $\alpha$-bi-Lipschitz homeomorphism $h: Y \rightarrow X$, and equicontinuous families $\left\{J_{y}\right\}_{y \in Y}$ and $\left\{G_{y}\right\}_{y \in Y}$ of real-linear (resp. linear) bijections from $E$ onto $F$ such that

$$
S f(y)=J_{y}(f(h(y))), \quad T f(y)=G_{y}(f(h(y)))
$$

for all $f \in \operatorname{Lip}_{\alpha}^{b}(X, E)$ and $y \in Y$.

Proof: Since for $\operatorname{Lip}_{\alpha}^{b}(X, E)\left(\operatorname{resp} . \operatorname{Lip}_{\alpha}^{b}(Y, F)\right)$ every point of $X$ (resp. $Y$ ) is a zero point it follows that $X_{0}=X$ and $Y_{0}=Y$, where $X_{0}, Y_{0}$ are subsets defined in Theorem 3.1. It is also clear that $E_{y}=E$ and $F_{y}=F$ for all $y \in Y$. Hence, by Theorem 3.1, for each $y \in Y$, there exist additive bijections $J_{y}, G_{y}: E \rightarrow F$ such that

$$
S f(y)=J_{y}(f(h(y))), \quad T f(y)=G_{y}(f(h(y)))
$$

for all $f \in \operatorname{Lip}_{\alpha}^{b}(X, E)$ and $y \in Y$.

We note that $S$ (and $T$ ) are $\mathbb{Q}$-homogeneous, that is, for each $r \in \mathbb{Q}$ and $x \in X, S(r x)=r S(x)$. An argument similar to [2, Corollaries 5.11 and 5.12] shows that $S$ has a closed graph. Now since $S$ is additive and $\mathbb{Q}$-homogeneous one can check easily that (as in the Closed Graph theorem, see, e.g., [10]) $S$ is continuous. Similarly $T$ is continuous. Hence $T$ and $S$ are, indeed, real-linear continuous maps which, in particular, yield the real-linearity of $J_{y}$ and $G_{y}$ for all $y \in Y$. Therefore, there exists $M>0$ such that

$$
\|S f\| \leq M\|f\| \quad\left(f \in \operatorname{Lip}_{\alpha}^{b}(X, E)\right) .
$$


Now for every $e \in E$ and $f \in \operatorname{Lip}_{\alpha}^{b}(X)$, let $f \otimes e \in \operatorname{Lip}_{\alpha}^{b}(X, E)$ be defined by $(f \otimes e)(x)=f(x) e$. Then

$$
\left\|J_{y}(e)\right\|=\|S(1 \otimes e)(y)\| \leq M\|e\| \quad(y \in Y, e \in E),
$$

which shows that the family $\left\{J_{y}\right\}$ of real-linear maps is equicontinuous. To finish the proof it suffices to show that $h$ and $k$ are $\alpha$-Lipschitz functions. Since by the above argument $S^{-1}$ is continuous, the family $\left\{J_{y}^{-1}\right\}$ of real-linear maps is equicontinuous and so there exists $N>0$ such that

$$
\left\|J_{y}^{-1}(\mathbf{f})\right\| \leq N\|\mathbf{f}\| \quad(y \in Y, \mathbf{f} \in F) .
$$

Fix a point $e_{0} \in E$ with $\left\|e_{0}\right\|=1$ and for each $y \in Y$ let $f_{y} \in \operatorname{Lip}_{\alpha}^{b}(X, E)$ be defined by $f_{y}(x)=d(x, h(y)) e_{0}, x \in X$. Using (3.1) we have $\left\|S f_{y}\right\| \leq$ $M\left\|f_{y}\right\| \leq M K$, where $K=\max \left\{\operatorname{diam}(X), \operatorname{diam}(X)^{1-\alpha}\right\}$. In particular $L_{\alpha}\left(S f_{y}\right) \leq M\left\|f_{y}\right\| \leq M K$ and for each $y^{\prime} \neq y$,

$$
\frac{d\left(h(y), h\left(y^{\prime}\right)\right)}{d^{\alpha}\left(y, y^{\prime}\right)}=\frac{1}{\left\|J_{y}\left(e_{0}\right)\right\|} \frac{\left\|S f_{y}(y)-S f_{y}\left(y^{\prime}\right)\right\|}{d^{\alpha}\left(y, y^{\prime}\right)} \leq N L_{\alpha}\left(S f_{y}\right) \leq N M K .
$$

Therefore, $\sup \frac{d\left(h(y), h\left(y^{\prime}\right)\right)}{d^{\alpha}\left(y, y^{\prime}\right)} \leq N M K$, where the supremum is taken over all distinct points $y, y^{\prime} \in Y$. Therefore $h: Y \rightarrow X$ satisfies the Lipschitz condition of order $\alpha$. Analogously it can be shown that $k$ is an $\alpha$-Lipschitz function.

Remark. (i) The above result is also valid for the case where $\operatorname{Lip}_{\alpha}^{b}(X, E)$ and $\operatorname{Lip}_{\alpha}^{b}(Y, F)$ are replaced by $\operatorname{lip}_{\alpha}^{b}(X, E)$ and $\operatorname{lip}_{\alpha}^{b}(X, E)$ respectively.

(ii) Boundedness of the metric spaces $X$ and $Y$ cannot be removed from the above corollary. Indeed, if $X$ is the discrete metric space $\mathbb{N}$, $Y$ is $\mathbb{N}$ equipped with the Euclidean metric, and $E=F=\mathbb{C}$, then clearly $\operatorname{Lip}_{\alpha}^{b}(Y, F)=l^{\infty}$ and, by [21, Example 1.6.4], $\operatorname{Lip}_{\alpha}^{b}(X, E)=l^{\infty}$. Now the identity operator $T: \operatorname{Lip}_{\alpha}^{b}(X) \rightarrow \operatorname{Lip}_{\alpha}^{b}(Y)$ preserves common zeros while $T$ is not continuous and also $X$ and $Y$ are not Lipschitz homeomorphic.

For a metric space $X$ and a normed space $E$, let $C_{b}^{u}(X, E)$ be the normed space of all bounded uniformly continuous functions from $X$ into $E$, equipped with the sup norm. Then clearly each point of $X$ is a zero point for $C_{b}^{u}(X, E)$.

Corollary 3.4. If $T, S: C_{b}^{u}(X, E) \rightarrow C_{b}^{u}(Y, F)$ are surjective linear maps which jointly preserve common zeros, then

$$
S f(y)=J_{y}(f(h(y))), \quad T f(y)=G_{y}(f(h(y)))
$$

for all $f \in C_{b}^{u}(X, E)$ and $y \in Y$, for some uniform homeomorphism $h: Y \rightarrow X$, and linear bijections $J_{y}, G_{y}: E \rightarrow F, y \in Y$. 
Proof: The description of $T, S$ is immediate from Theorem 3.1. Using the fact that $f \circ h \in C_{b}^{u}(Y)$ whenever $f \in C_{b}^{u}(X)$, and the argument given in [16, Theorem 2.3] and the remark after it, one can show that $h$ is uniformly continuous.

Remark. In the above corollary one can check easily that $S$ is continuous if and only if $\sup _{y \in Y}\left\|J_{y}\right\|<\infty$ and in this case $\|S\|=\sup _{y \in Y}\left\|J_{y}\right\|$. In particular, if $E$ or $F$ is finite dimensional, then $T$ and $S$ are continuous.

For a completely regular space $X$ and a Hausdorff topological vector space $E, C_{b}(X, E)$ denotes the space of all bounded continuous functions from $X$ into $E$.

Corollary 3.5. Suppose that $X$ and $Y$ are completely regular spaces consisting of $G_{\delta}$-points, and $E$ and $F$ are Hausdorff topological vector spaces. Let $T, S$ be surjections between the following spaces preserving jointly common zeros.

Case 1. T, $S: C(X, E) \rightarrow C(Y, F)$.

Case 2. T, $S: C_{b}(X, E) \rightarrow C_{b}(Y, F)$.

Then there exist a homeomorphism $h: Y \rightarrow X$, the bijections $J_{y}, G_{y}$ : $E \rightarrow F$ for each $y \in Y$, such that

$$
S f(y)=S 0(y)+J_{y}(f(h(y))), \quad T f(y)=T 0(y)+G_{y}(f(h(y))) .
$$

Furthermore, if $S, T$ are linear and $E$ or $F$ are finite dimensional, then $T$ and $S$ are continuous when the spaces in Cases 1 and 2 are equipped with the compact-open topology and sup norm topology, respectively.

Proof: Let $x \in X$ and take a sequence $\left\{V_{n}\right\}$ of the neighborhoods of $x$ such that $\{x\}=\cap_{n=1}^{\infty} V_{n}$. Since $X$ is a completely regular space, given $n \in \mathbb{N}$, there exists $f_{n} \in C_{b}(X)$ with $0 \leq f_{n} \leq 1, f_{n}(x)=1$, and $f_{n}=0$ on $X \backslash V_{n}$. Then letting $f=\left(1-\sum_{n=1}^{\infty} \frac{f_{n}}{2^{n}}\right) e$, where $e$ is a fixed nonzero element in $E$, we have $f \in C_{b}(X, E)$ with $Z(f)=\{x\}$. Clearly $C_{b}(X, E)$ and $C_{b}(Y, F)$ are completely regular, hence it follows from Corollary 3.2 that there exist a homeomorphism $h: Y \rightarrow X$ and bijections $J_{y}, G_{y}: E \rightarrow F$ for each $y \in Y$ such that

$$
S f(y)=S 0(y)+J_{y}(f(h(y))), \quad T f(y)=T 0(y)+G_{y}(f(h(y)))
$$

for all $f \in C_{b}^{u}(X, E)$ and $y \in Y$. In particular, if $S, T$ are linear then all bijections $J_{y}, G_{y}$ are linear. The continuity of $S, T$ is obtained by applying a similar argument as in [1, Corollary 4.2]. 
Remark. (i) Note that all points in a first countable completely regular space are $G_{\delta}$-points. But the converse is not true, that is there is a completely regular space consisting of $G_{\delta}$-points which is not first countable (see $[\mathbf{1 2}, 4 \mathrm{M}])$.

(ii) The following example borrowed from [11, Example 2.4] shows that in the above results when $E$ (and so $F$ ) is not a finite dimensional space, common zeros preserving maps need not be continuous (see also $[\mathbf{1}, \operatorname{Remarks}(2)])$.

Let $X=Y=\{0\}, \psi$ be a discontinuous linear functional of $c_{0}$ such that $\psi((1,0, \ldots))=1$ and define $J: c_{0} \rightarrow c_{0}$ by $J(\alpha)=\left(\alpha_{1}+\right.$ $\left.\psi(\alpha), \alpha_{2}, \alpha_{3}, \ldots\right)$ for all $\alpha=\left(\alpha_{1}, \alpha_{2}, \alpha_{3}, \ldots\right)$. It is clear that

$$
\left\{\begin{array}{l}
T: C\left(X, c_{0}\right) \rightarrow C\left(Y, c_{0}\right) \\
T f(0)=J(f(0))
\end{array}\right.
$$

is a discontinuous linear bijection which preserves common zeros.

\section{Jointly common zeros preserving maps between Banach modules}

In this section, using the notion of point multipliers, we introduce the notion of zero set for the elements of a Banach module $\mathcal{X}$ over a Banach algebra $\mathcal{A}$ with $\sigma(\mathcal{A}) \neq \emptyset$, and then we give a module version of Theorem 3.1 for mappings preserving common zeros between certain Banach modules.

We recall that $\nu_{\mathcal{A}}: \sigma_{\mathcal{A}}(\mathcal{X}) \rightarrow \sigma(\mathcal{A}) \cup\{0\}$ is the natural map which sends each nontrivial point multiplier $\xi$ on $\mathcal{X}$ in the unit ball of $\mathcal{X}^{*}$ to the unique point $\nu_{\mathcal{A}}(\xi)=\varphi \in \sigma(\mathcal{A}) \cup\{0\}$ satisfying $\langle\xi, a \cdot x\rangle=\varphi(a)\langle\xi, x\rangle$ for all $a \in \mathcal{A}$ and $x \in \mathcal{X}$. Since each closed hyper maximal submodule $P$ of $\mathcal{X}$ is the kernel of some $\xi \in \sigma_{\mathcal{A}}(\mathcal{X})$, and clearly point multipliers with the same kernels have the same image under $\nu_{\mathcal{A}}$, we may also use the notation $\nu_{\mathcal{A}}(P)$ instead of $\nu_{\mathcal{A}}(\xi)$ for $\xi \in \sigma_{\mathcal{A}}(\mathcal{X})$ with $\operatorname{ker}(\xi)=P$.

Now consider the following equivalence relation on $\Delta_{\mathcal{A}}(\mathcal{X})$. For $P, Q \in$ $\Delta_{\mathcal{A}}(\mathcal{X}), P \sim Q$ iff $\nu_{\mathcal{A}}(P)=\nu_{\mathcal{A}}(Q)$. There are some examples of left Banach modules $\mathcal{X}$ in which the equivalence classes are singleton. For instance, for a compact Hausdorff space $K$, the Banach $C(K)$-module $C(K)^{*}$ has this property [3, p. 317].

Definition 4.1. For an element $x \in \mathcal{X}$, we define the zero set of $x$ by $\mathcal{Z}(x)=\left\{\varphi \in \nu_{\mathcal{A}}\left(\sigma_{\mathcal{A}}(\mathcal{X})\right) \backslash\{0\}:\langle\xi, x\rangle=0\right.$ for all $\left.\xi \in \nu_{\mathcal{A}}^{-1}\{\varphi\}\right\}$.

We should note that there exists a nontrivial point multiplier on $\mathcal{X}$ at 0 if and only if $\mathcal{A} \cdot \mathcal{X}$ is not dense in $\mathcal{X}$. The definition shows that for 
each $x \in \mathcal{X}$, a nonzero point $\varphi$ in the range of $\nu_{\mathcal{A}}$ is in $\mathcal{Z}(x)$ if and only if $x \in P^{\prime}$ for all $P^{\prime} \in[P]$ where $P \in \nu_{\mathcal{A}}^{-1}(\varphi)$ is arbitrary and $[P]$ is its equivalence class.

If $\mathcal{A}$ is unital then, considering $\mathcal{A}$ as a Banach module over itself, we have $\sigma_{\mathcal{A}}(\mathcal{A})=\{\lambda \varphi: \varphi \in \sigma(\mathcal{A}), 0<|\lambda| \leq 1\}$ and so the defined zero set $\mathcal{Z}(a)$ of an element $a \in \mathcal{A}$ is the usual zero set of its Gelfand transformation $\hat{a}$ as a function on $\sigma(\mathcal{A})$.

Clearly for a compact plane set $K$ and uniformly closed subalgebra $\mathcal{A}$ of $C(K)$ which contains the constants and separates the points of $K$, the subspace $\mathcal{A} f$ of $C(K)$ for any non-vanishing function $f \in C(K)$ is a Banach $\mathcal{A}$-module. It is easy to see that the range of $\nu_{\mathcal{A}}$ is $\sigma(\mathcal{A})$. Since $f \in \mathcal{A} f$, for each nontrivial point multiplier $\xi$ on $\mathcal{A} f$ at some $\varphi \in \sigma(\mathcal{A})$ we have

$$
\langle\xi, a f\rangle=\varphi(a)\langle\xi, f\rangle \quad(a \in \mathcal{A})
$$

which shows that the zero set $\mathcal{Z}(a f)$ is the same as the usual zero set of $\hat{a}$.

The following examples show that the defined zero set may be very different according to the module action.

Example 4.2. For a compact Hausdorff space $K$ and a Banach space $E$, let $\mathcal{X}=C(K, E)$ be the Banach space of all continuous $E$-valued functions on $K$ endowed with the supremum norm. We also recall that for $e \in E$ and $f \in C(K), e \otimes f \in C(K, E)$ is defined by $(e \otimes f)(x)=$ $f(x) e, x \in K$. Then clearly $C(K, E)$ is a Banach $C(K)$-module and for each $\Lambda \in E^{*}, \Lambda \circ \varphi_{x}$, where $\varphi_{x}$ is the evaluation functional on $C(K)$ at $x \in K$, is a point multiplier at $\varphi_{x}$. On the other hand, for each point multiplier $\xi$ at some $\varphi_{x}, x \in K$, since by the proof of Lemma 1 in [13] the linear span of $\{e \otimes f: e \in E, f \in C(K)\}$ is dense in $C(K, E)$, it is easy to see that the functional $\Lambda \in E^{*}$ defined by $\Lambda(e)=\langle\xi, e \otimes 1\rangle$ satisfies $\xi=\Lambda \circ \varphi_{x}$. Hence $\sigma_{C(K)}(\mathcal{X})=\left\{\Lambda \circ \varphi_{x}: \Lambda \in E_{1}^{*} \backslash\{0\}, x \in K\right\}$, where $E_{1}^{*}$ is the unit ball of $E^{*}$. Moreover $\nu_{C(K)}$ maps each $\Lambda \circ \varphi_{x}$ to $\varphi_{x}$, that is the range of $\nu_{C(K)}$ is $\sigma(C(K))$. Therefore, for a function $\mathrm{F} \in C(K, E)$ we have

$$
\mathcal{Z}(\mathrm{F})=\left\{\varphi_{x}:\left(\Lambda \circ \varphi_{x}\right)(\mathrm{F})=0 \text { for all } \Lambda \in E_{1}^{*}\right\}=\left\{\varphi_{x}: \mathrm{F}(x)=0\right\},
$$

that is the new defined zero set of each element of $C(K)$-Banach module $C(K, E)$ is its zero set in the usual sense.

Example 4.3. Let $\mathcal{A}$ be a commutative unital Banach algebra and $E$ be a left Banach $\mathcal{A}$-module with $\sigma_{\mathcal{A}}(E) \neq \emptyset$. Then for a compact Hausdorff space $K, C(K, E)$ is a Banach $\mathcal{A}$-module under the following module 
action:

$$
(a \cdot \mathrm{F})(x)=a \cdot \mathrm{F}(x) \quad(a \in \mathcal{A}, \mathrm{F} \in C(X, E), x \in X) .
$$

It is easy to see that under this module structure on $C(K, E)$, for every $x \in K$ and point multiplier $\xi$ on $E$ at some point $\varphi \in \sigma(\mathcal{A})$, $\eta=\xi \circ \varphi_{x}$ is a point multiplier on $C(K, E)$ at $\varphi$. Assume, in addition that $E$ is not hyper semisimple and the natural map $\nu_{\mathcal{A}}^{E}: \sigma_{\mathcal{A}}(E) \rightarrow \sigma(\mathcal{A}) \cup\{0\}$ for $E$, is onto $\sigma(\mathcal{A})$ (for instance every non-semisimple commutative unital Banach algebra satisfies these properties as a Banach module over itself). Then clearly the associated natural map for $C(K, E)$ is also onto $\sigma(\mathcal{A})$. Now for a non-zero $e_{0} \in \operatorname{Rad}_{\mathcal{A}}(E)$ and the constant function $\mathrm{F}=e_{0} \otimes 1$, the usual zero set $Z(\mathrm{~F})=\{x \in K: \mathrm{F}(x)=0\}$ of $\mathrm{F}$ is empty while its zero set $\mathcal{Z}(\mathrm{F})$ as an element of $C(K, E)$ is the whole $\sigma(\mathcal{A})$, since for each point multiplier $\xi$ on $E,\left\langle\xi, e_{0}\right\rangle=0$ and for each point multiplier $\eta$ on $C(K, E)$ its restriction $\left.\eta\right|_{E}$ is either zero or a nontrivial point multiplier on $E$.

In the main theorem of this section (Theorem 4.5) we assume that $\mathcal{A}$ is a Banach algebra with non-empty spectrum, $\mathcal{X}$ is a left Banach $\mathcal{A}$-module with $\sigma_{\mathcal{A}}(\mathcal{X}) \neq \emptyset$, satisfying the following property:

(H) $\mathcal{X}$ contains an element with empty zero set and for each $\varphi \in$ $\nu_{\mathcal{A}}\left(\sigma_{\mathcal{A}}(\mathcal{X})\right) \backslash\{0\}$, there exists $x \in \mathcal{X}$ such that $\mathcal{Z}(x)=\{\varphi\}$.

We also assume that $\mathcal{B}$ is a Banach algebra and $\mathcal{Y}$ is a left Banach $\mathcal{B}$-module satisfying similar conditions.

Here is an example of a Banach $\mathcal{A}$-module satisfying these conditions:

Example 4.4. For a compact metric space $K$ and a closed nonempty proper subset $F$ of $K$, let $\mathcal{A}=\{a \in C(K): a=0$ on $\mathrm{F}\}$ and $\mathcal{X}=$ $C(K)$ endowed with the supremum norm and with the multiplication as its module action. Identifying each $x \in K$ with its corresponding evaluation functionals $\varphi_{x}$, since $\sigma(\mathcal{A})=K \backslash F$ it is easy to see that $\nu_{\mathcal{A}}$ maps $\sigma_{\mathcal{A}}(\mathcal{X})$ onto $K \backslash F \cup\{0\}$ and for each $\xi \in \sigma_{\mathcal{A}}(\mathcal{X})$ with $\nu_{\mathcal{A}}(\xi) \neq 0$ there exists $x \in K \backslash F$ such that $\left.\xi\right|_{\mathcal{A}}=\left.\lambda \varphi_{x}\right|_{\mathcal{A}}$ for some $\lambda \in \mathbb{C}$. Clearly for each $f \in \mathcal{X}$ we have $\mathcal{Z}(f) \subseteq Z(f) \backslash F$. Moreover, for each $a \in \mathcal{A}$, we have $\mathcal{Z}(a)=Z(a) \backslash F$. Since for each $x \in K \backslash F$ there exists $f \in \mathcal{X}$ with $f=1$ on $F \cup\{x\}$ and $|f|<1$ on $K \backslash(F \cup\{x\})$ it follows that $\mathcal{Z}(1-f)=Z(1-f) \backslash F=\{x\}$. Therefore for each $x \in K \backslash F,\{x\}$ is the zero set (in the defined sense) of an element of $\mathcal{X}$. Obviously $\mathcal{X}$ contains an element with empty zero set. 
As before, we say that a pair of linear maps $S, T: \mathcal{X} \rightarrow \mathcal{Y}$ preserves jointly common zero sets if for two elements $x, y \in \mathcal{X}, \mathcal{Z}(x) \cap \mathcal{Z}(y) \neq \emptyset$ iff $\mathcal{Z}(S x) \cap \mathcal{Z}(T y) \neq \emptyset$. We note that the maps $S$ and $T$ with this property are automatically injective whenever $\mathcal{X}$ is hyper semisimple and $\mathcal{A} \cdot \mathcal{X}$ is dense in $\mathcal{X}$. Indeed, suppose that $x \in \mathcal{X}$ with $T x=0$. Since each $\varphi \in \nu_{\mathcal{A}}\left(\sigma_{\mathcal{A}}(\mathcal{X})\right)$ is nonzero, we can take, by property $(\mathrm{H}), x^{\prime} \in \mathcal{X}$ such that $\mathcal{Z}\left(x^{\prime}\right)=\{\varphi\}$. By the assumption, $\mathcal{Z}\left(S x^{\prime}\right) \cap \mathcal{Z}(T x) \neq \emptyset$ and so $\mathcal{Z}(x) \cap \mathcal{Z}\left(x^{\prime}\right) \neq \emptyset$. Hence $\varphi \in \mathcal{Z}(x)$, which implies that $x=0$ by hyper semisimplicity of $\mathcal{X}$ and the density of $\mathcal{A} \cdot \mathcal{X}$ in $\mathcal{X}$.

Before stating the theorem we should note that for each $x \in \mathcal{X}$ and $Q_{0} \in \Delta_{\mathcal{A}}(\mathcal{X}),(x+Q)_{Q \in\left[Q_{0}\right]}$ may be considered as an element $\left(x_{Q}+\right.$ $Q)_{Q \in \Delta_{\mathcal{A}}(\mathcal{X})}$ of $\underline{\mathcal{X}}$, where $x_{Q}=x$ for all $Q \in\left[Q_{0}\right]$ and $x_{Q}=0$ for the other points $Q \in \Delta_{\mathcal{A}}(\mathcal{X})$. Finally let us put $\dot{\Delta}_{\mathcal{A}}(\mathcal{X}):=\left\{P \in \Delta_{\mathcal{A}}(\mathcal{X})\right.$ : $\left.\nu_{\mathcal{A}}(P) \neq 0\right\}$ which is the same as $\Delta_{\mathcal{A}}(\mathcal{X})$ when $\mathcal{A} \cdot \mathcal{X}$ is dense in $\mathcal{X}$.

Theorem 4.5. Let $\mathcal{X}$ and $\mathcal{Y}$ satisfy property $(\mathrm{H})$, and let $S, T: \mathcal{X} \rightarrow \mathcal{Y}$ be surjective maps jointly preserving common zeros. Then there exist a bijection $\tilde{h}: \dot{\Delta}_{\mathcal{B}}(\mathcal{Y}) / \sim \rightarrow \dot{\Delta}_{\mathcal{A}}(\mathcal{X}) / \sim$, submodules $E_{P}, F_{P}$ of $\underline{\mathcal{X}}$ and $\underline{\mathcal{Y}}$, respectively, and linear bijections $J_{P}, G_{P}: E_{P} \rightarrow F_{P}, P \in \dot{\Delta}_{\mathcal{B}}(\mathcal{Y})$, such that

$$
\begin{aligned}
& \left(S x+P^{\prime}\right)_{P^{\prime} \in[P]}=J_{P}\left((x+Q)_{Q \in \tilde{h}([P])}\right), \\
& \left(T x+P^{\prime}\right)_{P^{\prime} \in[P]}=G_{P}\left((x+Q)_{Q \in \tilde{h}([P])}\right),
\end{aligned}
$$

for all $x \in \mathcal{X}$ and $P \in \dot{\Delta}_{\mathcal{B}}(\mathcal{Y})$.

Proof: We prove the theorem through the following steps:

Step 1: Let $\varphi_{0} \in \nu_{\mathcal{A}}\left(\sigma_{\mathcal{A}}(\mathcal{X})\right) \backslash\{0\}$. Then there exists a unique point $\psi_{0} \in$ $\sigma(\mathcal{B})$ such that $\mathcal{Z}(S x)=\mathcal{Z}(T x)=\left\{\psi_{0}\right\}$ for all $x \in \mathcal{X}$ with $\mathcal{Z}(x)=\left\{\varphi_{0}\right\}$.

Let $x \in \mathcal{X}$ such that $\mathcal{Z}(x)=\left\{\varphi_{0}\right\}$. Then, by hypothesis, $\mathcal{Z}(T x) \neq \emptyset$. Assume, contrary to the claim in Step 1, that there exist two distinct points $\psi_{1}, \psi_{2} \in \mathcal{Z}(T x)$. Then there exists $b \in \mathcal{B}$ such that $\psi_{1}(b)=1$ and $\psi_{2}(b)=0$. Then for all $\xi_{1} \in \nu_{\mathcal{B}}^{-1}\left(\psi_{1}\right)$ and $\xi_{2} \in \nu_{\mathcal{B}}^{-1}\left(\psi_{2}\right)$ we have $\left\langle\xi_{1}, T x\right\rangle=\left\langle\xi_{2}, T x\right\rangle=0$. By hypotheses there exists $y_{0} \in \mathcal{Y}$ with $\mathcal{Z}\left(y_{0}\right)=$ $\emptyset$. Setting $s=b \cdot y_{0}$ and $t=y_{0}-s$ we get elements $s, t \in \mathcal{Y}$ such that

$$
\left\langle\xi_{2}, s\right\rangle=\left\langle\xi_{2}, b \cdot y_{0}\right\rangle=\psi_{2}(b)\left\langle\xi_{2}, y_{0}\right\rangle=0,
$$

that is, $\psi_{2} \in \mathcal{Z}(s)$. In particular, $\mathcal{Z}(s) \neq \emptyset$ and so $\mathcal{Z}\left(s^{\prime}\right) \neq \emptyset$, where $s^{\prime}$ is an element in $\mathcal{X}$ with $S s^{\prime}=s$. Since $\psi_{2} \in \mathcal{Z}(s) \cap \mathcal{Z}(T x)$ it follows that $\mathcal{Z}\left(s^{\prime}\right) \cap \mathcal{Z}(x) \neq \emptyset$ and therefore $\varphi_{0} \in \mathcal{Z}\left(s^{\prime}\right)$. On the other hand,

$$
\left\langle\xi_{1}, t\right\rangle=\left\langle\xi_{1}, y_{0}\right\rangle-\psi_{1}(b)\left\langle\xi_{1}, y_{0}\right\rangle=0
$$


that is $\psi_{1} \in \mathcal{Z}(t) \cap \mathcal{Z}(T x)$. Now taking $t^{\prime} \in \mathcal{X}$ with $S t^{\prime}=t$ we deduce that $\mathcal{Z}\left(t^{\prime}\right) \cap \mathcal{Z}(x) \neq \emptyset$, i.e. $\varphi_{0} \in \mathcal{Z}\left(t^{\prime}\right)$. Therefore $\varphi_{0} \in \mathcal{Z}\left(s^{\prime}\right) \cap \mathcal{Z}\left(t^{\prime}\right)$ and consequently $\mathcal{Z}\left(S s^{\prime}\right) \cap \mathcal{Z}\left(T t^{\prime}\right) \neq \emptyset$, therefore we can find an element $\xi \in \mathcal{Z}\left(S s^{\prime}\right) \cap \mathcal{Z}\left(T t^{\prime}\right)=\mathcal{Z}(s) \cap \mathcal{Z}(t)$ which clearly satisfies $\xi(s+t)=$ $\xi\left(y_{0}\right)=0$, a contradiction.

Similarly there is a unique point in $\mathcal{Z}(S x)$. Furthermore $\mathcal{Z}(S x)=$ $\mathcal{Z}(T x)$ since $\mathcal{Z}(S x) \cap \mathcal{Z}(T x) \neq \emptyset$.

We should note that for each $\varphi \in \nu_{\mathcal{A}}\left(\sigma_{\mathcal{A}}(\mathcal{X})\right) \backslash\{0\}$, the unique point in $\mathcal{Z}(T x)$ does not depend on the element $x \in \mathcal{X}$ with $\mathcal{Z}(x)=\{\varphi\}$, since for each $x^{\prime} \in \mathcal{X}$ with $\mathcal{Z}\left(x^{\prime}\right)=\{\varphi\}$ we have clearly $\mathcal{Z}(S x) \cap \mathcal{Z}\left(T x^{\prime}\right) \neq \emptyset$ and $\mathcal{Z}(T x) \cap \mathcal{Z}\left(S x^{\prime}\right) \neq \emptyset$.

Using the step above we can associate to each $\varphi \in \nu_{\mathcal{A}}\left(\sigma_{\mathcal{A}}(\mathcal{X})\right) \backslash\{0\}$ the unique point $k(\varphi) \in \nu_{\mathcal{B}}\left(\sigma_{\mathcal{B}}(\mathcal{Y})\right) \backslash\{0\}$ such that $\mathcal{Z}(T x)=\mathcal{Z}(S x)=$ $\{k(\varphi)\}$, where $x$ is an element of $\mathcal{X}$ with $\mathcal{Z}(x)=\{\varphi\}$.

Similarly, for each $\psi \in \nu_{\mathcal{B}}\left(\sigma_{\mathcal{B}}(\mathcal{Y})\right) \backslash\{0\}$, we can find a unique point $h(\psi) \in \nu_{\mathcal{A}}\left(\sigma_{\mathcal{A}}(\mathcal{X})\right) \backslash\{0\}$ such that for each $y \in \mathcal{Y}$ with $\mathcal{Z}(y)=\{\psi\}$, we have $\mathcal{Z}(x)=\mathcal{Z}\left(x^{\prime}\right)=\{h(\psi)\}$, where $x, x^{\prime}$ are elements in $\mathcal{X}$ with $S x=T x^{\prime}=y$.

Step 2: For each $x \in \mathcal{X}, k(\mathcal{Z}(x)) \subseteq \mathcal{Z}(T x) \cap \mathcal{Z}(S x)$ and $h(\mathcal{Z}(T x)) \cup$ $h(\mathcal{Z}(S x)) \subseteq \mathcal{Z}(x)$.

Let $\varphi_{0} \in \mathcal{Z}(x)$ and let $x_{0}$ be such that $\mathcal{Z}\left(x_{0}\right)=\left\{\varphi_{0}\right\}$, by property $(\mathrm{H})$. Then clearly $\mathcal{Z}(T x) \cap \mathcal{Z}\left(S x_{0}\right) \neq \emptyset$ and $\mathcal{Z}(S x) \cap \mathcal{Z}\left(T x_{0}\right) \neq \emptyset$. Therefore $k\left(\varphi_{0}\right) \in \mathcal{Z}(T x) \cap \mathcal{Z}(S x)$.

A similar argument implies the other inclusion.

Step 3: The maps $h$ and $k$ are bijective, in fact $k(h(\varphi))=\varphi$ and $\psi=$ $h(k(\psi))$ for each nonzero $\varphi$ and $\psi$ in the range of $\nu_{\mathcal{B}}$ and $\nu_{\mathcal{A}}$, respectively.

Assume first that $h\left(k\left(\varphi_{0}\right)\right) \neq \varphi_{0}$ for some $\varphi_{0} \in \nu_{\mathcal{A}}\left(\sigma_{\mathcal{A}}(\mathcal{X})\right) \backslash\{0\}$. Then setting $\varphi_{1}=h\left(k\left(\varphi_{0}\right)\right)$ we can find $a \in \mathcal{A}$ such that $\varphi_{0}(a)=0$ and $\varphi_{1}(a)=1$. Let $s=a \cdot x_{0}$, where $x_{0} \in \mathcal{X}$ is such that $\mathcal{Z}\left(x_{0}\right)=\left\{\varphi_{0}\right\}$. Then for each $\xi_{0} \in \nu_{\mathcal{A}}^{-1}\left(\varphi_{0}\right)$ we have

$$
\left\langle\xi_{0}, s\right\rangle=\varphi_{0}(a)\left\langle\xi_{0}, x_{0}\right\rangle=0
$$

that is $\varphi_{0} \in \mathcal{Z}(s)$. Hence by Step $2, k\left(\varphi_{0}\right) \in \mathcal{Z}(T s)$ and using Step 2 once again we have $\varphi_{1}=h\left(k\left(\varphi_{0}\right)\right) \in \mathcal{Z}(s)$ while since $\varphi_{1} \notin \mathcal{Z}\left(x_{0}\right)$ there exists $\xi_{1} \in \nu_{\mathcal{A}}^{-1}\left(\varphi_{1}\right)$ such that $\left\langle\xi_{1}, x_{0}\right\rangle \neq 0$ and therefore

$$
\left\langle\xi_{1}, s\right\rangle=\varphi_{1}(a)\left\langle\xi_{1}, x_{0}\right\rangle \neq 0
$$


which is impossible. Hence $h\left(k\left(\varphi_{0}\right)\right)=\varphi_{0}$. Similarly $k(h(\psi))=\psi$ for all nonzero $\psi$ in the range of $\nu_{\mathcal{B}}$.

We now define $\tilde{h}: \dot{\Delta}_{\mathcal{B}}(\mathcal{Y}) / \sim \rightarrow \dot{\Delta}_{\mathcal{A}}(\mathcal{X}) / \sim$ by $\tilde{h}([P])=[Q]$, where for each $P \in \dot{\Delta}_{\mathcal{B}}(\mathcal{Y}), Q \in \dot{\Delta}_{\mathcal{A}}(\mathcal{X})$ is an arbitrary element in $\nu_{\mathcal{A}}^{-1}\left(h\left(\nu_{\mathcal{B}}(P)\right)\right)$. Clearly $\tilde{h}$ is well-defined and bijective.

For $P \in \dot{\Delta}_{\mathcal{B}}(\mathcal{Y})$ consider the following subsets of $\underline{\mathcal{X}}$ and $\underline{\mathcal{Y}}$ :

$$
\begin{aligned}
E_{P} & :=\left\{(x+Q)_{Q \in \tilde{h}([P])}: x \in \mathcal{X}\right\}, \\
F_{P} & :=\left\{\left(y+P^{\prime}\right)_{P^{\prime} \in[P]}: y \in \mathcal{Y}\right\} .
\end{aligned}
$$

Then $E_{P}$ and $F_{P}$, which are subsets of $\underline{\mathcal{X}}$ and $\mathcal{Y}$ respectively, are submodules of these modules.

Now define the maps $J_{P}, G_{P}: E_{P} \rightarrow F_{P}$ by $J_{P}\left((x+Q)_{Q \in \tilde{h}([P])}\right)=$ $\left(S x+P^{\prime}\right)_{P^{\prime} \in[P]}$ and $G_{P}\left((x+Q)_{Q \in \tilde{h}([P])}\right)=\left(T x+P^{\prime}\right)_{P^{\prime} \in[P]}$. Using the definition of $\mathcal{Z}(\cdot)$ and Step 2, we see that $J_{P}$ and $G_{P}$ are well-defined.

Step 4: For each $P \in \dot{\Delta}_{\mathcal{B}}(\mathcal{Y}), J_{P}$ and $G_{P}$ are linear bijections.

It is clear that for each $P \in \dot{\Delta}_{\mathcal{B}}(\mathcal{Y}), J_{P}$ and $G_{P}$ are linear and surjective. Assume now that $J_{P}\left((x+Q)_{Q \in \tilde{h}([P])}\right)=0$, that is $S x \in P^{\prime}$ for all $P^{\prime} \in[P]$. Hence $\nu_{B}(P) \in \mathcal{Z}(S x)$ and, by Step $2, h\left(\nu_{\mathcal{B}}(P)\right) \in \mathcal{Z}(x)$. Therefore, $x \in Q$ for all $Q \in \tilde{h}([P])$, i.e. $(x+Q)_{Q \in \tilde{h}([P])}=0$ and so $J_{P}$ (similarly, $G_{P}$ ) is injective.

Proposition 4.6. Under the hypotheses of Theorem 4.5, if furthermore, $\mathcal{A}, \mathcal{B}$ are regular and the zero sets of elements in $\mathcal{X}, \mathcal{Y}$ are all closed, then the map $h$ given by this theorem is a homeomorphism.

Proof: It suffices to show that $h$ is continuous since the same holds for $k$. Assume on the contrary that there exists a net $\left\{\varphi_{\alpha}\right\}$ in $\nu_{\mathcal{B}}\left(\sigma_{\mathcal{B}}(\mathcal{Y})\right) \backslash\{0\}$ converging to a point $\varphi_{0} \in \nu_{\mathcal{B}}\left(\sigma_{\mathcal{B}}(\mathcal{Y})\right) \backslash\{0\}$ such that $h\left(\varphi_{\alpha}\right)$ does not converge to $h\left(\varphi_{0}\right)$. Therefore, passing to a subnet, we can assume that there exists a neighborhood $U$ of $h\left(\varphi_{0}\right)$ such that $h\left(\varphi_{\alpha}\right) \notin U$ for all $\alpha$. Hence by the regularity of $\mathcal{A}$ we can find an element $a \in \mathcal{A}$ such that $h\left(\varphi_{0}\right)(a)=1$ and $h\left(\varphi_{\alpha}\right)(a)=0$ for all $\alpha$. Hence setting $x=a \cdot x_{0}$, where $x_{0} \in \mathcal{X}$ with $\mathcal{Z}\left(x_{0}\right)=\emptyset$, we get an element $x$ in $\mathcal{X}$ such that for each $\alpha$ and $\xi \in \nu_{\mathcal{A}}^{-1}\left(h\left(\varphi_{\alpha}\right)\right)$ we have

$$
\langle\xi, x\rangle=\left\langle\xi, a \cdot x_{0}\right\rangle=h\left(\varphi_{\alpha}\right)(a)\left\langle\xi, x_{0}\right\rangle=0,
$$

that is, $h\left(\varphi_{\alpha}\right) \in \mathcal{Z}(x)$ for all $\alpha$. Hence by Step 2, $\varphi_{\alpha}=k\left(h\left(\varphi_{\alpha}\right)\right) \in$ $\mathcal{Z}(T x)$ for all $\alpha$. Therefore, $\varphi_{0} \in \mathcal{Z}(T x)$ since $\mathcal{Z}(T x)$ is assumed to be closed. Again Step 2 yields $h\left(\varphi_{0}\right) \in \mathcal{Z}(x)$, which is impossible, since $\mathcal{Z}\left(x_{0}\right)=\emptyset$ and $h\left(\varphi_{0}\right)(a)=1$. 
Acknowledgements. We would like to thank the referees for their invaluable comments and suggestions.

\section{References}

[1] J. Araujo, Realcompactness and Banach-Stone theorems, Bull. Belg. Math. Soc. Simon Stevin 11(2) (2004), 247-258.

[2] J. Araujo And L. Dubarbie, Biseparating maps between Lipschitz function spaces, J. Math. Anal. Appl. 357(1) (2009), 191-200. DOI : $10.1016 /$ j.jmaa. 2009.03.065.

[3] J. BRAČıč, Simple multipliers on Banach modules, Glasg. Math. J. 45(2) (2003), 309-322. DOI: 10.1017/S0017089503001265.

[4] J. CaO, I. Reilly, And H. Xiong, A lattice-valued BanachStone theorem, Acta Math. Hungar. 98(1-2) (2003), 103-110. DOI: 10.1023/A: 1022861506272.

[5] D. Chen, L. Li, R. Wang, and Y.-S. Wang, Nonvanishing preservers and compact weighted composition operators between spaces of Lipschitz functions, Abstr. Appl. Anal. 2013 (2013), Article ID 741050, 8 pages. DOI: 10.1155/2013/741050.

[6] J. X. Chen, Z. L. Chen, And N.-C. Wong, A Banach-Stone theorem for Riesz isomorphisms of Banach lattices, Proc. Amer. Math. Soc. 136(11) (2008), 3869-3874. DOI: 10.1090/S0002-993908-09582-8.

[7] L Dubarbie, Maps preserving common zeros between subspaces of vector-valued continuous functions, Positivity 14(4) (2010), 695-703. DOI : 10.1007/s11117-010-0046-z.

[8] Z. ERCAN AND S. ÖnAL, Banach-Stone theorem for Banach lattice valued continuous functions, Proc. Amer. Math. Soc. 135(9) (2007), 2827-2829. DOI: 10.1090/S0002-9939-07-08788-6.

[9] Z. ERCAN AND S. ÖNAL, The Banach-Stone theorem revisited, Topology Appl. 155(16) (2008), 1800-1803. DOI: 10.1016/j.topol. 2008.05.018.

[10] G. B. Folland, "Real analysis. Modern techniques and their applications", Second edition, Pure and Applied Mathematics (New York), A Wiley-Interscience Publication, John Wiley \& Sons, Inc., New York, 1999.

[11] H.-L. Gau, J.-S. Jeang, And N.-C. Wong, Biseparating linear maps between continuous vector-valued function spaces, J. Aust. Math. Soc. 74(1) (2003), 101-109. DOI: 10.1017/S1446788700003153.

[12] L. Gillman AND M. JeRison, "Rings of continuous functions", The University Series in Higher Mathematics, D. Van Nostrand Co., Inc., Princeton, N.J.-Toronto-London-New York, 1960. 
[13] A. Hausner, Ideals in a certain Banach algebra, Proc. Amer. Math. Soc. 8 (1957), 246-249. DOI: 10.1090/S0002-9939-1957-0084117-4.

[14] K. JARosz, Automatic continuity of separating linear isomorphisms, Canad. Math. Bull. 33(2) (1990), 139-144. DOI: 10.4153/ CMB-1990-024-2.

[15] A. Jiménez-Vargas, A. Morales Campoy, And M. VilleGAS-VAlLECILlos, The uniform separation property and BanachStone theorems for lattice-valued Lipschitz functions, Proc. Amer. Math. Soc. 137(11) (2009), 3769-3777. DOI: 10.1090/S0002-993909-09941-9.

[16] M. Lacruz and J. G. Llavona, Composition operators between algebras of uniformly continuous functions, Arch. Math. (Basel) 69(1) (1997), 52-56. DOI: 10.1007/s000130050092.

[17] D. H. Leung And W.-K. TAng, Banach-Stone theorems for maps preserving common zeros, Positivity 14(1) (2010), 17-42. DOI: $10.1007 / \mathrm{s} 11117-008-0002-3$.

[18] L. Li AND N.-C. Wong, Banach-Stone theorems for vector valued functions on completely regular spaces, J. Math. Anal. Appl. 395(1) (2012), 265-274. DOI: 10.1016/j.jmaa.2012.05.033.

[19] L. Li And N.-C. Wong, Kaplansky Theorem for completely regular spaces, Proc. Amer. Math. Soc. 142(4) (2014), 1381-1389. DOI: 10.1090/S0002-9939-2014-11889-2.

[20] X. Miao, J. CaO, And H. Xiong, Banach-Stone theorems and Riesz algebras, J. Math. Anal. Appl. 313(1) (2006), 177-183. DOI: 10.1016/j.jmaa.2005.08.050.

[21] N. Weaver, "Lipschitz algebras", World Scientific Publishing Co., Inc., River Edge, NJ, 1999. DOI : 10.1142/4100.

Maliheh Hosseini:

Department of Mathematics

K. N. Toosi University of Technology

Tehran, 16315-1618

Iran

E-mail address: m.hosseini@kntu.ac.ir

Fereshteh Sady:

Department of Pure Mathematics

Faculty of Mathematical Sciences

Tarbiat Modares University

Tehran, 14115-134

Iran

E-mail address: sady@modares.ac.ir

Primera versió rebuda el 16 de març de 2015, darrera versió rebuda el 22 d'octubre de 2015. 